\title{
Bologna, deleghe e pratiche composite
}

Patrizia Gabellini

Il testo di Patrizia Gabellini discute il ruolo dello spazio, il modo di 'giocare' la competenza tecnica, la possibilità di intercettare la cultura locale, continuità e discontinuità, riferendosi alle diverse deleghe assessorili: urbanistica, ambiente e città storica. 'Resistenza' (alle richieste di mettere in gioco nuove aree urbanizzabili), 'Emergenza' (da affrontare per l'interruzione dei cantieri e i fallimenti aziendali), 'Innovazione' (per aprire altre prospettive di intervento) sono $i$ termini coi quali distingue e connota le scelte fatte in campo urbanistico. 'Emersione' (portando fuori dal cono d'ombra questioni relegate alla discussione in ambienti già sensibilizzati) e 'Integrazione' (di politiche e azioni che scuotono il settorialismo tecnico e amministrativo) identificano, rispettivamente, le scelte ambientali e per il centro storico. I casi descritti da Francesco Evangelisti consentono di approfondire le implicazioni dell'Emergenza e dell'Integrazione

Parole chiave: urbanistica; amministrazione; competenza

\section{Bologna: Different strategies Concerning the Management of the Crisis}

In her text, Patrizia Gabellini discusses the role of space, the means of 'playing' with technical skill, the possibility of intercepting local culture, and continuity $v s$. discontinuity, with reference to her different administrative powers. Resistance (to requests to include new urbanizable areas), Innovation (opening of different perspectives regarding intervention), and Emergency (solutions to face crises in suspended works) are terms that distinguish the choices made in the field of urban planning. Emergence (from the shadow of questions relegated to discussion in already sensitized fields) and Integration (of policies and actions that upset the sectoral nature of techniques and administration) identify, respectively, choices for the environment and the historical city centre. The cases described by Francesco Evangelisti investigate the implications of Emergency and Integration

Keywords: urbanism; urban management; competence
Politecnico di Milano, Dipartimento di Architettura e Studi Urbani (patrizia.gabellini@polimi.it)

\section{Deleghe}

Il combinato delle deleghe assessorili è molto importante, anche se solitamente sottaciuto. Ho deciso di mettere l'accento su questo aspetto, scegliendolo come titolo del testo, in quanto il ruolo dello spazio, il modo di 'giocare' la competenza (Pizzorno, Crosta, Secchi, 2013; De Leo, Forester, 2016), la possibilità di intercettare la cultura locale sui temi urbani e territoriali si sono dati in maniera diversa nei tre principali ambiti della mia attività di assessore nel Comune di Bologna: quello proprio dell'urbanistica (comprensivo dell'edilizia privata), quelli dell'ambiente e della città storica. Li richiamerò con riferimento alle intenzioni di cui ho caricato il mio lavoro, ad alcuni esiti argomentabili, a qualche valutazione e congettura sul futuro.

Ciascuno di questi campi di intervento ha una propria storia alle spalle, non solo locale, con altrettante questioni aperte che sono state stressate dallo straordinario cambiamento in atto, emerso con indiscussa evidenza negli anni del mandato amministrativo. Assolvere ai compiti richiesti dalla responsabilità attribuitami con queste deleghe non mi ha posto solo specifici dilemmi, da trattarsi in maniera apposita, ma ha anche (ovviamente) generato intersezioni tra le scelte che andavo facendo e inciso sulla strutturazione del discorso che quelle scelte ha accompagnato, inducendomi a cercare correlazioni che dessero un senso a pratiche tra loro distanti. Il mio modo di intendere la resilienza, per esempio (Gabellini, 2013c; 2014a), e il mio interesse per le aperture del recycle (Gabellini, 2015a; 2017b) nascono proprio da questo incontro tra mondi che, progressivamente, ha reso per me consueto intersecare differenti piani operativi e riflessivi.

L'argomento col quale l'allora candidato sindaco Virginio Merola mi chiese di andare a ricoprire quel ruolo era centrato sull'urbanistica, e verteva sostanzialmente sull'idea che potessi occuparmi della gestione di un piano "che conoscevo meglio di lui» (ero stata consulente per la sua costruzione quando lui era Assessore all'urbanistica col Sindaco Sergio Cofferati) (Bonfantini, Evangelisti, 2009), alleggerendo il suo lavoro. Le altre deleghe non erano al centro del nostro patto di collaborazione e ne venni a conoscenza in seguito, anzi, quelle alla Città storica e alla Qualità urbana mi furono rese note poco prima della presentazione ufficiale della Giunta alla città. Questo incipit mi sembra connotare un'esperienza che, nel tempo, si è confermata tripartita.

Il primo pacchetto di deleghe comprendeva anche la Qualità urbana e fin dall'inizio l'onere mi era parso pesante, ma coglievo l'importanza di collocare il mio lavoro in quella prospettiva, 


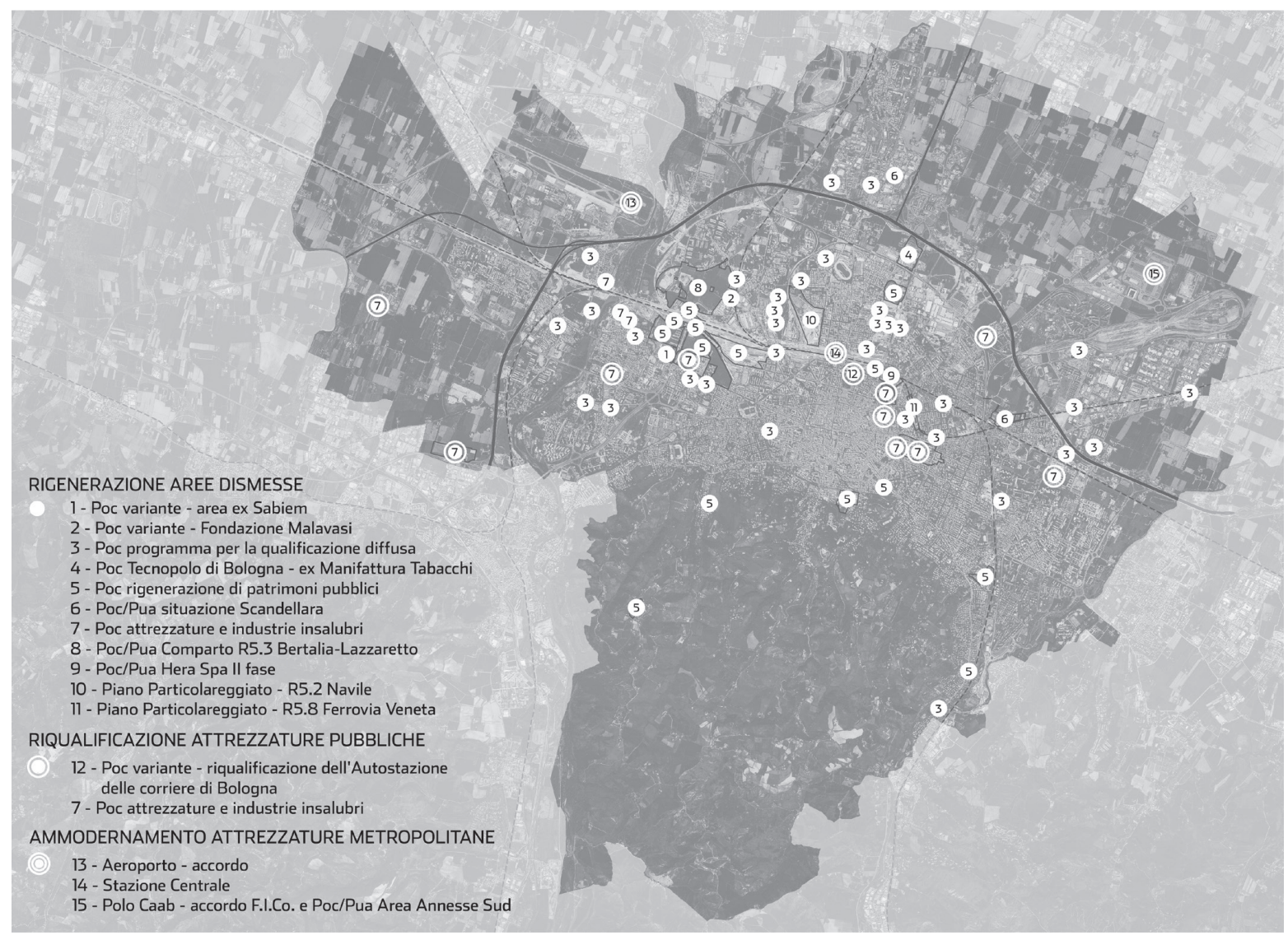

Mappa della rigenerazione

Fonte: Settore Piani e progetti urbanistici, Comune di Bologna

comunemente connessa con le competenze urbanistiche (qualità urbana è espressione la cui genesi va ampiamente ascritta ai nostri ambienti culturali). L'accostamento con le deleghe per Urbanistica e Ambiente, ma soprattutto con quella per la Città storica, creava connessioni interessanti benché gravide di possibili conseguenze. La qualità urbana, infatti, rinvia allo spazio, ma nello stesso tempo è espressione vaga e ampiamente inclusiva in termini di oggetti, competenze, fenomeni da considerare, mettendo in gioco anche aspetti sfuggenti come la percezione 'comune'. Qualità urbana è un costrutto teorico che cela un grumo di questioni assai complesse da distinguere concettualmente, quasi imprendibile se lo si voglia trattare in maniera efficace dal punto di vista amministrativo (può funzionare come messaggio politico), soprattutto richiede un tempo di lavoro difficilmente compatibile con quello veloce e occasionale della percezione, poco o per nulla sensibile a dati e argomenti fino a quando non si 'vedono' cambiamenti nelle cose.

Occuparmi di qualità urbana ha comportato una consistente dilatazione della materia da curare, evidente nelle domande che mi venivano poste in Consiglio comunale al question time settimanale: abbandono dei rifiuti, imbrattamento dei muri e uso improprio degli spazi pubblici con comportamenti scorretti di vario genere (dal consumo di droghe alla presenza di persone senza fissa dimora), quindi questioni strettamente legate alle politiche sociali e tangenti la sicurezza, anzi, via via più strettamente legate a quest'ultima. L'istituzione di una 'Cabina di regia' per combattere il degrado da un lato (nella quale l'assessore coinvolto non ero io), il carico di lavoro dall'altro, mi hanno indotto, in occasione della verifica di metà mandato, a rimettere la delega nelle mani del Sindaco, il solo a mio avviso che potesse avere il controllo dell'intera tastiera di responsabilità coinvolte dal tema.

Tuttavia, due anni e mezzo di convivenza con questa delega mi hanno consentito di individuare la cosiddetta 'mappa del degrado', diversa in ogni città e che, a Bologna, porta in evidenza alcune aree più esterne dove si trovano edifici dismessi e spazi irrisolti, ma anche aree centrali dove si trova il nucleo storico delle sedi universitarie e parchi urbani dalla morfologia complessa e/o collocati in contesti socialmente difficili. La mappa di una 'periferia arcipelago' che ancora oggi si scontra con la radicata idea di un centro geografico e 'storico', dove si presume una concentrazione della qualità urbana, opposto a una 
periferia geografica e 'moderna', dove si presume la sua carenza o assenza totale, mentre stenta ad affermarsi la consapevolezza del degrado come fenomeno composito e dinamico, entro il quale lo spazio gioca un ruolo parziale e specifico da identificare ogni volta.

\section{Profili}

Oltre alla composizione delle deleghe e alle ragioni per cui il Sindaco ha deciso di affidarle, a mio avviso costituiscono sfondo indispensabile per inquadrare un'esperienza amministrativa, il modo di interpretare il ruolo (Gabellini, 2017a) e il profilo della città.

A Bologna non c'era l'esigenza di fare un piano o di costruire una diversa/nuova cornice per le politiche urbane, bensì quella di riprendere un discorso che era stato interrotto per circa due anni caratterizzati da un mandato amministrativo breve e dissonante poi dal commissariamento, eventi traumatici per la città e per il Partito democratico (Vogelij, 2015). Si trattava di riprenderlo con scelte utili per affrontare i problemi in parte diversi che si affollavano sul tavolo dell'amministrazione, considerando anche la distanza che la sopravvenuta crisi aveva creato tra il piano impostato nei primi anni Duemila e la situazione all'inizio del secondo decennio. Consideravo tutto ciò assai impegnativo, tanto più in una città che, forte del suo passato, sull'urbanistica non faceva sconti. Ne è seguita una full immersion, per cui mi è sembrato indispensabile chiedere l'aspettativa all'università decretando la 'sospensione' di tutte le attività connesse, benché il movente fondamentale dell'accettazione del nuovo incarico fosse la ricerca. Infatti, riconosco come ragione di fondo della mia scelta la curiosità per i cambiamenti e le dinamiche che muovono una città, la cui conoscenza comporta a mio avviso quegli «incontri ravvicinati del terzo tipo» (sceneggiati da Spielberg) che la pratica amministrativa consente. Potrei aggiungere, con altro riferimento anomalo, che riconoscevo quella come un'occasione unica per entrare nel «ventre della città». Quindi, l'esperienza della routine che si associa al ruolo come veicolo imprescindibile, e la disponibilità a muovermi nelle pieghe della vita amministrativa, assieme a un'interpretazione non rigida dei confini di competenza. Questo (forse anche) per il mio rapporto particolare con Bologna, città dove ho scelto di risiedere all'inizio degli anni '70, di cui ho scritto, dove ho lavorato a più riprese: non la 'mia' città, ma quella con cui ho maturato una particolare consuetudine ed empatia.

Bologna è città dall'immagine forte nella quale spiccano il 'buon governo' della sinistra che l'ha amministrata senza soluzione di continuità per oltre mezzo secolo (dal 1945 al 1999, quando si costituisce la prima giunta di centro-destra), il benessere connesso a un'economia articolata e al dinamismo del capitale sociale, la presenza dell'Università e di un ingente patrimonio storico tramandato grazie a un singolare impegno conservativo. Oggi città metropolitana di piccola taglia, assimilabile a poche altre realtà europee (Indovina, Fregolent, Savino, 2005; Gabellini, Proli, Tondelli, 2015). Questi tratti distintivi aiutano a interpretare quel che dirò, anche quando sposterò l'attenzione sulla dimensione 'ordinaria', non unica ed esemplare, dei temi dominanti (è significativo che Virginio Merola in più occasioni abbia rivendicato il suo essere un sindaco 'normale'). Mi riferirò dunque a quel che si è fatto o si è cercato di fare rispetto alle questioni che più, oggi, accomunano Bologna ad altre città italiane medio-grandi, sempre considerando l'ombra lunga di un pregresso e le condizioni ineludibili di un contesto.

\section{Urbanistica: resistenza}

Per quanto riguarda l'urbanistica, mi sembra di poter riassumere il lavoro fatto in tre mosse: a) opporre resistenza alle richieste di mettere in gioco nuove aree urbanizzabili, b) fronteggiare l'emergenza creata dall'interruzione dei cantieri e dai fallimenti aziendali, c) aprire altre prospettive di intervento.

All'inizio del mandato si è imposta subito la scelta che ho indicato come resistenza: 'congelare' le nuove urbanizzazioni comprese nel Piano strutturale, esito di una successione di decisioni maturate negli anni della grande crescita, poi discusse, rielaborate e ridimensionate nello stesso Psc e arrivate alla crisi senza che vi fossero riconosciuti i diritti edificatori. Infatti, per la Lr. 20/2000 e la successiva 6/2009, questo avviene solo se le aree del Piano strutturale sono inserite nel Piano operativo comunale ed era ciò che mi veniva chiesto di fare e che per molti era considerato ovvio. Le condizioni erano però cambiate, come dimostravano chiaramente i mancati interventi nelle aree dismesse (anche in quelle inserite nel primo POC del 2009) e l'interruzione delle trasformazioni più significative.

Il confronto con i costruttori è stato ed è tuttora considerato 'il' nodo per un assessore all'urbanistica. Se nel condurlo va indubbiamente attivata la capacità di mediazione politica, è invece problematico il modo di utilizzare la competenza tecnica e di coniugarla (se coniugarla) con l'altra. Le fattispecie del connubio sono varie e si distinguono proprio per il loro posizionamento lungo l'asse che va da un approccio solo politico a uno solo tecnico. E solitamente l'esito viene discusso proprio in riferimento a questo posizionamento.

Io sono stata mossa dalla convinzione che, nella situazione data, non si dovessero aprire nuovi fronti, sia per la necessità di non consumare suolo agricolo e naturale, sia soprattutto per l'inevitabile competizione che si sarebbe generata rispetto alla trasformazione delle aree dismesse: l'eccedenza di offerta avrebbe probabilmente favorito le realizzazioni su suolo libero accentuando le difficoltà della rigenerazione. Mi animava la preoccupazione che si avviassero operazioni senza futuro, aggiungendo altri problemi a quelli già evidenti. Oggi rileggo la mia posizione come coerente con quella definita delle «minime previsioni urbanistiche» negli anni '60 (Campos Venuti, 1967). Nel lungo confronto con gli operatori direttamente interessati e nelle diverse sedi (Giunta, Commissioni, Consiglio comunale, dibattiti pubblici) ho giocato la mia competenza per sottolineare le modifiche di scenario, evidenziare i rischi dell'incompiuto, contestare la finalità solo finanziaria delle richieste a fronte della mancanza di domanda (gli stessi operatori che chiedevano l'inserimento delle nuove aree nel POC avevano interrotto i loro cantieri in altre parti della città). Una resistenza argomentata che la crisi a catena delle imprese ha smesso di sollecitare dopo qualche anno (si sono quasi interrotti gli incontri con i rappresentanti dell'Associazione nazionale dei costruttori e della Lega delle cooperative edili), e che confidava sulla velocità dei processi in atto e sulla possibilità che essi modificassero le convenienze degli operatori rendendo anche ai loro occhi più conveniente investire altrimenti. Non ho ritenuto invece opportuno avviare una variante del PSC (assai laboriosa tecnicamente e certamente osteggiata), considerando prioritario concentrare il lavoro sui POC e sull'adeguamento del Regolamento urbanistico edilizio, oltre che necessario un quadro metropolitano per procedere in quella direzione. 
I segnali che vengono da altre realtà italiane sembrano dare ragione al mio 'temporeggiamento', d'altro canto, però, la diversa composizione degli attori in campo (modificata dal lato degli operatori e dei loro rappresentati nelle associazioni di categoria, ma anche dal lato delle istituzioni, comunali e sovralocali) strutturerà altrimenti il discorso, destinato a riaprirsi sia perché resta irrisolta la questione di una 'svalutazione governata' delle aree senza mercato, oltre a quella dei crediti bancari ad esse legati, sia perché al 'congelamento' nel Piano strutturale non conformativo corrisponde (ambiguamente) il pagamento di ICI/IMU (Gabellini, $2015 b$ ). Una nuova legge urbanistica regionale, ora in discussione, con un impianto profondamente diverso dalla precedente (al centro la rigenerazione e il contenimento del consumo di suolo, un unico piano urbanistico che non conferisce diritti edificatori e che si attua attraverso «accordi operativi» con gli operatori) e il piano territoriale della città metropolitana, cui è affidata la responsabilità di distribuire tra i comuni le quote di suolo 'consumabile', saranno a riguardo decisivi, anche perché aleggia in città una vaga aspettativa di ripresa e restano incerti gli orientamenti delle società di gestione dei fondi immobiliari e delle Ferrovie dello Stato, i due influenti proprietari delle grandi aree dismesse. A me resta la convinzione di avere opportunamente interrotto l'iter urbanistico che avrebbe portato al conferimento di diritti edificatori dai quali (eventualmente) recedere sarebbe stato difficile, se non altro a causa delle implicazioni giuridiche e delle possibili conseguenze economiche per l'Amministrazione. Constato, tuttavia, che l'idea di una 'città arcipelago', ossia di una forma urbana che alterna parti più o meno compatte con spazi aperti, anche agricoli, esito combinato di dispersione e shrinkage e presupposto di un progetto decisamente centrato sulla rigenerazione, incontra una straordinaria resistenza, anche culturale, se si esce da ristretti circoli di 'competenti' (Gabellini, 2015c).

\section{Urbanistica: emergenza}

I cantieri interrotti a causa delle crisi e dei fallimenti aziendali hanno creato situazioni di emergenza che richiedevano altre modalità di intervento, mettendo a dura prova una competenza urbanistica calibrata sulla 'distanza' temporale (tempi medio-lunghi) e spaziale (lo sguardo dall'alto). Di fronte all'inceppamento di operazioni sulle quali si era scommesso per la trasformazione urbana, come l'area dell'ex Mercato ortofrutticolo (Ginocchini, Tartari, 2007), ho misurato i limiti di una tecnica che non contempla la crisi della domanda né la velocità del cambiamento, e che oggi non trova agganci in un progetto economico e sociale, quindi politico, per ridefinirsi compiutamente dovendo navigare a vista. In queste situazioni ci si deve confrontare con uno spazio che non prende forma o potrà prenderla a fatica in un tempo non programmabile, mentre gli abitanti delle parti realizzate e della zona che gravita attorno all'area irrisolta ritengono un loro diritto avere un orizzonte temporale definito e vivere in uno spazio risolto. I cantieri abbandonati diventano, invece, epifenomeni di un'incertezza spaziale e temporale insopportabile, le cui implicazioni per un'amministrazione vanno ben aldilà di una probabile perdita di consenso e fiducia: vi si giocano le sorti urbanistiche e la coesione sociale di interi settori urbani. Negli incontri, numerosi e difficili, di tipo assembleare o riservati ai rappresentanti dei Comitati, ho fatto (anche in questo caso) uno sforzo argomentativo orientato alla 'trasparenza', cercando di motivare le ragioni per le quali lo spazio avrebbe preso forma lentamente e con aggiustamenti forse rilevanti rispetto al progetto originario (quello 'partecipato' di cui molti erano stati protagonisti e che aveva convinto altri a comprare lì piuttosto che altrove), per cui occorreva trovare e accettare soluzioni parziali, di miglioramento pro-tempore e/o come ponte verso altre più incisive. Con la rimozione delle macerie accatastate, la ripulitura, una 'primaria' sistemazione dei grandi spazi rimasti vuoti, potevano risolversi le principali difficoltà (mancanza di illuminazione, di percorsi sicuri e di ombra, di recinzioni dignitose) consentendo il presidio dell'area via frequentazione e fermando l'inevitabile scivolamento verso il degrado. Si è dunque deciso di anticipare l'intervento pubblico (che avrebbe dovuto pagarsi con gli oneri di urbanizzazione derivanti dalla vendita e trasformazione delle aree di proprietà comunale presenti nel comparto), da me sostenuto non solo quale necessità per rendere abitabile in modo civile l'area, ma anche quale possibile innesco per riavviare il progressivo completamento attirando investimenti privati. Attraverso un accurato lavoro di approfondimento economico e giuridico da parte degli uffici, è stato messo a punto un programma di interventi diretti dell'Amministrazione comunale, tali da completare le principali opere di sistemazione dello spazio pubblico, mentre l'utilizzo di varie fonti di finanziamento, compresa quella aperta con il Piano città, consentiva di finanziare le attrezzature pubbliche lì concentrate per servire un'ampia porzione del quartiere Navile (come descritto da Evangelisti a p. 47).

I lavori di sistemazione a terra sono quasi completati, gli alberi piantati cresceranno e daranno forma allo spazio attenuando l'impressione di desolazione. I cantieri delle opere pubbliche dureranno alcuni anni e c'è qualche segnale di interesse da parte di nuovi investitori. Questo mi fa ritenere che la decisione presa sia stata non solo ragionevole, ma anche strategica per casi simili, portando in primo piano la necessità di un ragionamento approfondito sul ruolo dell'intervento pubblico in questa fase di stallo, quando, invece, attenzione e ricerca sono polarizzati sull'incentivazione dell'investimento privato. Inoltre, l'evidente rigidità dei progetti urbani concepiti per essere realizzati in maniera unitaria e in pochi anni suggerisce di cambiare strada rivisitando approcci, rimasti nell'ombra, volti a elaborare progetti realizzabili per parti disetanee ma in sé compiute, di considerare e progettare la convivenza con i cantieri, di accettare il carattere tentativo delle decisioni, di andare a fondo sugli usi temporanei. Le tante dimensioni del 'piano processo' si impongono nell'esperienza comune.

In questo caso la competenza è entrata in gioco per l'abitudine a cercare analogie e suggerimenti in altre esperienze, a mettere insieme i tasselli, a immaginare come il singolo tassello potrebbe modificare lo spazio delineato nel progetto/piano di partenza con slittamenti interessanti, a scegliere gli strumenti da mettere in campo, a modificare alcune regole. Cruciale in queste situazioni è l'interpretazione del disegno urbanistico e dei suoi margini di modificabilità.

\section{Urbanistica: innovazione}

L'attività che più si presta a connotare il mandato è però legata al modo di utilizzare i piani operativi. In 5 anni ne sono stati redatti e approvati 3 di respiro generale, concepiti con l'intenzione di aprire realistiche possibilità di intervento in una città 'inceppata', focalizzandosi sulla rigenerazione e forzando le maglie 
della legge regionale là dove i POC sono strumenti generalisti di attuazione del piano strutturale (Gabellini, 2014c).

Il primo, dal titolo Piano operativo di qualificazione diffusa, può considerarsi una 'mossa del cavallo', laddove ha accettato la richiesta di non bloccare del tutto un'attività immobiliare già asfittica, ma ha aperto una strada diversa da quella attesa, spingendo verso la riqualificazione di aree e immobili di piccola e media taglia, abbandonati o in via di abbandono all'interno delle parti strutturate del territorio urbanizzato. Ciò ha comportato la selezione degli ambiti nei quali indirizzare e sollecitare gli interventi, l'appello rivolto a precisi interlocutori (i proprietari e non le imprese e gli operatori immobiliari) invitati a partecipare manifestando il loro interesse a intervenire, la individuazione (con approvazione consiliare) dei requisiti per la selezione delle cento domande raccolte con la call, incontri di vaglio/aggiustamento delle proposte tramite tavoli misti, con tecnici dei vari settori comunali e amministratori dei quartieri, un puntiglioso lavoro di affinamento tecnico-giuridico-amministrativo per costruire convenzioni nelle quali, per la prima volta, si usavano dispositivi atti a ottenere quali dotazioni anche opere 'minori' di sistemazione degli spazi pubblici esistenti o la monetizzazione di piccole quote di edilizia residenziale sociale (il $20 \%$ stabilito dalla legge, in interventi di dimensioni contenute, produce poche unità di alloggi, se non addirittura improbabili porzioni).

Si è indubbiamente trattato dell'operazione più consonante con quell'idea di riqualificazione diffusa associata alla manutenzione e cura della città che, per un breve lasso di tempo, è stata indicata dagli urbanisti come alternativa alla fine della stagione caratterizzata dal 'grande progetto urbano'. Operazione in grado di farci comprendere la grana estremamente varia della riqualificazione, le conseguenti soglie della dotazione pubblica e il ruolo talvolta indispensabile della monetizzazione (con i relativi problemi di gestione del 'salvadanaio'), il rapporto tra prestazione e valutazione, l'intersezione con le politiche energetiche e ambientali: tutti aspetti che presuppongono confidenza con la forma e l'organizzazione dello spazio.

A me sembra questo l'atto con il più alto tasso di tecnicalità, persino creativo, eppure in grado di entrare nel circuito della comunicazione pubblica e con un'evidente valenza politica per la sua capacità di intercettare una domanda di azione (non si può solo frenare e rimediare) e di orientamento politico e amministrativo, non di semplice transizione. Considerando anche i cantieri che piano piano si sono aperti e si aprono nelle aree incluse nel Piano (una trentina), individuo questo POC come una sorta di manifesto della mia delega urbanistica e mi sento di riproporre alcune considerazioni fatte a questo riguardo nel 2012, all'avvio dell'operazione:

«L'esperienza che ho tratteggiato (in corso e in parte tentativa), consapevolmente centrata sulla trasformazione diffusa e la manutenzione, può incidere profondamente sul volto e le pratiche d'uso della città, ma è un'attività in sordina che richiede tempo e aggiustamenti continui, che delude le domande radicali. [...] Se sullo sfondo si staglia ormai nettamente un nuovo paradigma per le politiche urbane e territoriali, nell'immediato si impone una successione di azioni dal carattere modesto e processuale, disposte su piani diversi, affidate all'adesione diffusa e alla costanza nel tempo» (Gabellini, 2014b: 138).

Eppure grandi operazioni di rigenerazione sono necessarie, anche se la crisi le ha allontanate nel tempo. A Bologna riguar- dano le aree demaniali e ferroviarie e sono determinanti per la ristrutturazione urbana.

Una successione impegnata e faticosa di accordi interistituzionali sottoscritti con i soggetti proprietari (Demanio dello Stato, Cassa depositi e prestiti, Ferrovie dello Stato, Università di Bologna, Invimit-SGR) doveva trovare una formalizzazione prima della chiusura del mandato: non solo c'erano le condizioni indispensabili, ma si trattava anche di decisione dall'evidente carattere strategico rispetto al Piano strutturale e alla volontà di creare $\mathrm{i}$ presupposti affinché centinaia di ettari abbandonati e inquinati, collocati nel bel mezzo della città, venissero 'prima o poi' riusati, interrompendo il circolo vizioso determinato dalla loro presenza. In quel prima o poi che ho evidenziato si situa il problema: l'incertezza degli sviluppi possibili a fronte di una domanda al momento assente o indefinita si scontrava con i caratteri operativi dello strumento che avevamo a disposizione e, per contro, l'indeterminatezza del Piano strutturale rispetto ai diritti bloccava l'iniziativa dei soggetti interessati, in particolare l'impegno dei fondi ministeriali, unica risorsa all'orizzonte. Si trattava di una scommessa politica per l'Amministrazione e di una scommessa tecnica, che si è giocata nella particolare caratterizzazione dei documenti del POC rigenerazione dei patrimoni pubblici (anche il più 'duro' di questi come la Valsat), piegati per corrispondere all'incertezza dei tempi di realizzazione (Comune di Bologna, 2015).

\section{Ambiente: emersione}

I tratti distintivi del lavoro fatto nel campo ambientale mi sembrano riassumibili, da un lato, nell'utilizzo consapevole di un discreto grado di autonomia decisionale, dall'altro, nell'impegno a de-settorializzare politiche e azioni.

Come ho detto, la delega ambientale non era all'origine dei motivi per i quali il Sindaco aveva ritenuto di coinvolgermi, né è usuale combinarla con quella all'urbanistica. L'abbinamento era stato una scelta dell'Amministrazione precedente, opportunamente reiterata. Interessante era la presenza di una struttura tecnica articolata, già coinvolta nella costruzione del Piano strutturale e attrezzata per partecipare (con successo) a significativi bandi europei con finanziamento di progetti innovativi. Nonostante, o forse grazie alla fatica di dover entrare nel merito dei tanti differenti temi (dai rifiuti all'amianto, dal verde all'acqua, dall'inquinamento dell'area a quello del suolo, dal rumore delle attività temporanee a quello determinato dalla presenza dell'aeroporto, dall'energia al clima), comportante applicazione e studio, ho potuto comprendere appieno la dimensione multisensoriale dello spazio e la necessità di elaborare proposte attente non solo alla componente visibile, ma anche a quella sonora, tattile e olfattiva della città, e mi sono convinta che da lì si poteva partire per ridare spessore a tecniche e politiche urbanistiche. Da questo punto di vista tutte le questioni che arrivavano sul mio tavolo, in toto o marginalmente (alcune coinvolgevano anche altri assessori), mi apparivano meritevoli di essere approfondite e trattate, tecnicamente coinvolgenti e politicamente ineludibili. Benché implicassero competenze che avevano ciascuna una solida base scientifica e tecnica che non possedevo, emergevano le assonanze con quella urbanistica e prospettavano intersezioni fertili con evidenti ricadute sulla forma e sull'organizzazione dello spazio, verso quella che è stata indicata come «EcoWebTown» (Clementi, 2016). 
Considerati in maniera aggregata, $\mathrm{i}$ temi ambientali non erano centrali per l'agenda politica della maggioranza consiliare, però lo erano singolarmente in relazione alla loro incidenza sulla vita quotidiana dei cittadini. Si è determinata una situazione, solo apparentemente paradossale, per cui alcuni temi erano dominanti nelle tante segnalazioni dei cittadini cui dovevo rispondere giorno per giorno e conseguentemente impegnavano Giunta, Consiglio e forze politiche, oltre a occupare le pagine locali dei giornali, eppure alcuni degli atti più significativi per le loro implicazioni immediate e il futuro della città, come il Piano d'azione per l'energia sostenibile e il Piano di adattamento ai cambiamenti climatici (Barbi, Fini, Gabellini, 2016; Gabellini, 2016), o per l'individuazione delle priorità di investimento e spesa e la valutazione delle politiche, come il Bilancio ambientale, apparivano specialistici e lasciati nel cono d'ombra. Per cercare di colmare questo disallineamento tra contingenza e strategia, ho ritenuto fondamentale portare a compimento regolamenti e piani (anche quello per la rimozione dell'amianto, a seguito di un'impegnativa Istruttoria pubblica) sottoponendoli, nelle diverse fasi dell'iter, all'attenzione della Commissione consiliare e del Consiglio comunale, senza sottrarmi a tutte le occasioni di dibattito (anche se solitamente poco frequentate): nei quartieri, nei circoli o nelle feste del Partito democratico.

Consideravo e considero tuttora un vantaggio lavorare senza essere al centro dell'attenzione, come invece capitava per altri temi, per portare a termine attività tecniche assai esigenti in termini di impegno e tempo. La predisposizione degli strumenti di pianificazione, infatti, costituisce un supporto non secondario per l'integrazione delle competenze e può rimuovere alcuni ostacoli all'azione, spingendo in avanti le situazioni. Benché non vi fosse necessità di avviare un percorso amministrativo analogo a quello previsto per gli strumenti urbanistici, ho ritenuto necessario farlo per allargare l'area del confronto agli organi istituzionali, utilizzando le sedute pubbliche per portare l'attenzione su questioni altrimenti relegate alla discussione in ambienti già sensibilizzati. Ora Bologna si è candidata come European Green Capital 2019, avvalendosi anche dei piani e progetti approvati che costituiscono una sorta di credenziale. Anche in questo caso un lavoro continuo e in sordina, con alcune uscite pubbliche, a volte impegnative, che mettono opportunamente in tensione l'habitus tecnico e l'approccio settoriale.

\section{Città storica: integrazione}

Anche la delega per la città storica ha messo in luce il nodo dell'interazione/integrazione, investendo in maniera altrettanto rilevante il piano amministrativo e quello tecnico. Mi limiterò a considerare le scelte fatte per il centro storico, cui abbiamo deciso di dedicare un affondo col testo di Evangelisti data l'indiscussa centralità: per il rapporto con il programma elettorale del Sindaco, per l'ampio coinvolgimento degli assessori, per la sensibilità dei Bolognesi, per l'attenzione dei media, queste scelte possono ritenersi distintive della prima Giunta Merola. Di nuovo in centro. Programma per la pedonalità a Bologna (Prospero, 2012; Evangelisti, 2012; Gabellini, 2013a) riassume i tratti salienti della politica per il centro storico e costituisce un caso interessante anche per i temi coi quali si confronta. A Bologna il centro è una 'città nella città' (per il numero di abitanti residenti, la gamma degli usi e il funzionamento complessivo), caratterizzata da un insediamento e una presenza studentesca tali da indurre l'Università nella tentazione di concepirlo come proprio campus, interessata da consistenti fenomeni di trasformazione in parte legati al processo di metropolizzazione. Il tutto concorre a creare un ambiente urbano con un'alta complessità delle pratiche sociali.

L'impostazione e l'avvio del programma, già nei primi mesi del mandato, sono stati contraddistinti da una decisa tensione innovativa, e il livello raggiunto nella sua realizzazione è stato rilevante per le risorse di bilancio impiegate, l'entità ed estensione degli interventi (assai noti i 'TDays', ovvero la pedonalizzazione estesa nei fine settimana), gli effetti sull'economia urbana che solo in parte si mostrano nel 'volto' sensibilmente modificato del centro storico.

La sistemazione dello spazio è sotto gli occhi di tutti, è quel visibile che costruisce la 'prima' immagine della città. Molti meccanismi che presiedono al suo funzionamento, invece, sfuggono allo sguardo e rinviano a processi tecnici e burocratici, sociali ed economici difficili da identificare precisamente e da cogliere in tutte le loro implicazioni. Del programma, quindi, non mi sembra tanto significativo rilevare le iniziali opposizioni, da parte di commercianti e taxisti soprattutto (con dinamiche già riconosciute in casi simili), quanto sottolineare alcune conseguenze della sua caratteristica trasversalità, anche sul ruolo del mio Assessorato.

I tanti interventi che Di nuovo in centro ha promosso, o cui ha fatto da cornice, hanno messo in tensione le relazioni tra Assessorati e tra apparati, posti per la prima volta di fronte alle implicazioni di una politica urbana che cerca di coniugare regolazione della mobilità (non mera pedonalizzazione), riqualificazione dello spazio pubblico (dai sottoservizi, agli usi, agli arredi, alla gestione), valorizzazione delle attività economiche, coinvolgimento attivo degli abitanti e dei gestori delle attività commerciali e artigianali. Se nella fase di costruzione del programma, col sostegno del Settore piani e progetti urbanistici, ho potuto assumere il compito di comporre le istanze e di valorizzare il carattere multiplo della policy utilizzando la mia conoscenza urbanistica, nella fase di progettazione-appalto-esecuzione dei singoli interventi e di attivazione delle molteplici azioni connesse, anche di gestione dei conflitti, quando il coordinamento diventava per certi versi essenziale, sono 'rientrata' nei limiti delle mie deleghe, ritenendo che la legittimazione del coordinamento potesse venire solo da un'investitura politica che poco aveva a che fare con la mia competenza.

A me sembra che le difficoltà incontrate nel costruire un discorso 'corale', amministrativo e tecnico, non siano solo da ascriversi alla distanza tra la sfida aperta (opportuna e interessante come dimostra il riconoscimento venuto col Premio Gubbio 2015), l'organizzazione complessiva della 'macchina' comunale che ancora fatica con la logica dell' integrazione, e il mancato coordinamento tra gli assessori. Mi sembra che, almeno in questa sede, si debba notare che mettere insieme assetti spaziali e politiche urbane comporta la costruzione di un delicato rapporto tra differenti abiti mentali e pratiche, quelli legati a progetti e norme da un lato, a dialogo e concertazione dall'altro, e che tutto ciò richiede lunga e costante applicazione oltre a salda convinzione. Poi il confronto con le tante, diverse e talvolta contrapposte componenti sociali coinvolte dal programma (residenti e abitanti temporanei, giovani e anziani, commercianti e ambulanti, eser- 
centi delle attività legate alla movida notturna, ciclisti, taxisti, azienda dei trasporti pubblici, associazioni ambientaliste e per la tutela storica, ...) ha messo a nudo le aporie della «democrazia di prossimità» (Ascher, 2005).

\section{Continuità e discontinuità}

È mio convincimento che il buon governo delle città abbia nella continuità una sua condizione e che la discontinuità debba avere ragioni fondate e argomentabili. Nel caso di Bologna, per quel che concerneva le mie deleghe, la necessità di cambiare non si poneva in assoluto, ma in maniera puntuale e diversificata. Il breve mandato amministrativo del Sindaco Delbono e la successiva gestione del Commissario Cancellieri avevano avviato o sospeso operazioni che dovevano essere singolarmente riprese e aggiustate, le mutate condizioni al contorno ponevano problemi nuovi.

Per l'urbanistica, la ricerca di soluzioni diverse a fronte dei problemi aperti dalla crisi è stata continua e disinibita e ha riguardato anche l'abbandono dell'impostazione che era stata data all'uso della perequazione urbanistica (riconoscimento dei diritti edificatori da trasferire senza prefigurarne luoghi e modi di 'atterraggio'). Una continuità sui generis può riconoscersi con la politica ambientale impostata negli anni ' 90 e proceduta in sordina con alcune timidezze: essa si è declinata come ripresa, congiunzione tra componenti che apparivano isolate, messa in valore e inserimento a pieno titolo nel circuito amministrativo. Per il centro storico, ho preso atto di una polarizzazione del discorso e degli interventi su questa parte della città che aveva sostanzialmente ignorato le implicazioni teoriche e operative dell'idea di 'città storica' introdotta dal Piano strutturale e dal Regolamento urbanistico edilizio (Evangelisti, Orlandi, Piccinini, 2008; Gabellini, 2013b). Evidentemente difficile da cogliere in tutte le sue sfumature, quell'impostazione era sopravvissuta nel linguaggio, ma aveva perso gran parte del suo mordente. Poiché era indubbiamente emersa una questione 'centro storico', la mia preoccupazione principale è stata quella di mantenere o reintrodurre, là dove possibile, temi messi a fuoco entro una diversa concettualizzazione, benché la continuità con il mio lavoro di consulente non sia mai stato il presupposto o il pensiero dominante.

\section{Riferimenti bibliografici}

Ascher F., 2005, I nuovi principi dell'urbanistica, a cura di Russo M. Napoli: Tullio Pironti.

Barbi V., Fini G., Gabellini P., 2016, a cura di, Bologna città resiliente. Sostenibilità energetica e adattamento ai cambiamenti climatici/Bologna Resilient City. Sustainable Energy and Climate Change Adaptation. Bologna: Urban Center Bologna.

Campos Venuti G., 1967, Amministrare l'urbanistica. Torino: Einaudi.

Clementi A., 2016, Forme imminenti. Città e innovazione urbana. Rovereto: List.

Comune di Bologna, 2009, Bologna. Leggere il nuovo piano urbanistico $P S C+R U E+P O C$, a cura di Bonfantini B., Evangelisti F. Ferrara: Edisai.
Comune di Bologna - Dipartimento di riqualificazione urbana, Settore Piani e Progetti Urbanistici, UI Progettazione Urbanistica, 2015, POC rigenerazione di patrimoni pubblici. Bologna: Urban Center Bologna. Twww.urbancenterbologna.it (ultimo accesso 31/07/2017).

De Leo D., Forester J., 2016, «Italian Planning Overview and Perspectives Through Stories and Profiles. Notes from a Critical Pragmatist Research on Planning Practices». Crios, 2: 33-40. Doi: 10.3280/ CRIOS2016-012004.

Evangelisti F., 2012, «Di nuovo in centro. Una nuova pedonalità per Bologna». In: Piccinini M. (a cura di), «Viaggio in Italia. Bologna». Urbanistica Informazioni, 242: 51-3.

Evangelisti F., Orlandi P., Piccinini M., 2008, a cura di, La città storica contemporanea. Ferrara: Edisai.

Gabellini P., 2013a, a cura di, «I progetti per il centro di Bologna». Urbanistica Informazioni, 251: 59-71.

Gabellini P., 2013b, Vagliare il deposito della grande crescita. In: Albani F., Di Biase C. (a cura di), Architettura minore del XX secolo. Strategie di tutela e intervento. Sant'Arcangelo di Romagna: Maggioli.

Gabellini P., 2013c, «Rigenerazione e resilienza». In: Leonori M., Testa P. (a cura di), La città oltre lo sprawl. Rendita, consumo di suolo e politiche urbane ai tempi della crisi. Italianieuropei. Roma: Edizioni Solaris.

Gabellini P., 2014a, «La strada della resilienza». In Russo M., a cura di, Urbanistica per una diversa crescita. Progettare il territorio contemporaneo. Roma: Donzelli.

Gabellini P., 2014b, «Capire il carattere della crisi, agire gradualmente e selettivamente, accettare la parzialità: una crisi nella crisi». In: Fregolent L., Savino M. (a cura di), Città e politiche in tempi di crisi. Milano: FrancoAngeli. Gabellini P., 2014c, «Il piano di Bologna e il grande cambiamento». Inforum, 46: 4-6.

Gabellini P., 2015a, «Imbattersi nei rifiuti». In: Pavia R., Secchi R., Gasparrini C. (a cura di), Il territorio degli scarti e dei rifiuti, Re-Cycle Italy 08. Roma: Aracne Editrice.

Gabellini P., 2015b, «Temi della ricerca nel caso di Bologna». In: Urbani P. (a cura di), Politiche urbanistiche e gestione del territorio. Tra esigenze del mercato e coesione sociale. Torino: G. Giappichelli editore.

Gabellini P., 2015c, «Grandi questioni e rappresentazioni della città/Big questions about and representations of the city». Urbanistica, 156 (finito di stampare luglio 2017).

Gabellini P., 2016, «Il progetto BlueAp e il Piano di adattamento climatico di Bologna: un caso 'ordinario'». Sentieri urbani, 20: 22-24.

Gabellini P., 2017a, «La reinvenzione del proprio ruolo». In: De Leo D., L'urbanistica dei prof(ass) essori. Esperienze e competenze nell'amministrazione pubblica e per la didattica. Milano: FrancoAngeli.

Gabellini P., 2017b, «Re-cycle, ovvero rilavorare lo spazio urbanizzato». In: Fontanari E., Piperata G. (a cura di), Agenda Recycle. Proposte per reinventare la città. Bologna: il Mulino, 243-254.

Gabellini P., Proli S., Tondelli S., 2015, «Città metropolitana di Bologna». In: De Luca G., Moccia F.D. (a cura di), Immagini di territori metropolitani. Roma: Inu Edizioni (e-book).

Ginocchini G., Tartari C., 2007, a cura di, Il Mercato: una storia di rigenerazione urbana a Bologna. Ferrara: Edisai.

Indovina F., Fregolent L., Savino M., 2005, a cura di, L'esplosione della città. Catalogo della mostra. Bologna: Editrice Compositori.

Pizzorno A., Crosta P.L., Secchi B., 2013, Competenza e rappresentanza, a cura di Bianchetti C., Balducci A. Roma: Donzelli.

Prospero A., 2012, a cura di, Di nuovo in centro. Programma per la pedonalità a Bologna/A programme for a Bologna city centre pedestrian friendly. Bologna: Urban Center Bologna.

Vogelij J., 2015, «Effective Strategy Making. Co-Designing Scenarios as a Tool for Effective Strategic Planning». Architecture and the Built Environment, 15: 153-181. TU Delft. 